\title{
Optimization of exposure and countryside waste management for different accidental radioactive release
}

\author{
Philippe Guétat ${ }^{*}$ \\ Member of High Committee for Transparency and Information about Safety in Nuclear
}

\begin{abstract}
Since the Fukushima accident, Japanese scientists have been intensively monitoring ambient radiations in the highly contaminated territories situated within $80 \mathrm{~km}$ of the nuclear site. The surveys that were conducted through mainly carborne, airborne and in situ gamma-ray measurement devices, enabled to efficiently characterize the spatial distribution and temporal evolution of air dose rates induced by Caesium134 and Caesium-137 in the terrestrial systems. These measurements revealed that radiation levels decreased at rates greater than expected from physical decay in 2011-2012 (up to a factor of 2), and dependent on the type of environment (i.e. urban, agricultural or forest). Unlike carborne measurements that may have been strongly influenced by the depuration of road surfaces, no obvious reason can be invoked for airborne measurements, especially above forests that are known to efficiently retain and recycle radiocaesium.
\end{abstract}

The purpose of our research project is to develop a comprehensive understanding of the data acquired by Japanese, and identify the environmental mechanisms or factors that may explain such decays. The methodology relies on the use of a process-based and spatially-distributed dynamic model that predicts radiocaesium transfer and associated air dose rates inside/above a terrestrial environment (e.g., forests, croplands, meadows, bare soils and urban areas).

\begin{abstract}
Despite the lack of site-specific data, our numerical study predicts decrease rates that are globally consistent with both aerial and in situ observations. The simulation at a flying altitude of $200 \mathrm{~m}$ indicated that ambient radiation levels decreased over the first 12 months by about $45 \%$ over dense urban areas, $15 \%$ above evergreen coniferous forests and between 2 and 12\% above agricultural lands, owing to environmental processes that are identified and discussed. In particular, we demonstrate that the decrease over evergreen coniferous regions might be due the combined effects of canopy depuration (through biological and physical mechanisms) and the shielding of gamma rays emitted from the forest floor by vegetation. Our study finally suggests that airborne surveys might have not reflected dose rates at ground level in forest systems, which were predicted to slightly increase by 5 to $10 \%$ during the same period of time.
\end{abstract}

\footnotetext{
*Corresponding author: philippe.guetat $@$ cea.fr
} 
Optimization of exposure and waste management for different accidental releases

\author{
Philippe GUETAT \\ Stackholder - France
}

- Paris 2016 
- Multi-criteria analysis based on realities

- Protection: radiological and conventional

- Wealth : country and individuals

- Time, work and pleasure of living

- Definition of the boundary between:

- concentration + .........- dilution +

- confinement =>

- radioactive waste! ! ! no waste 


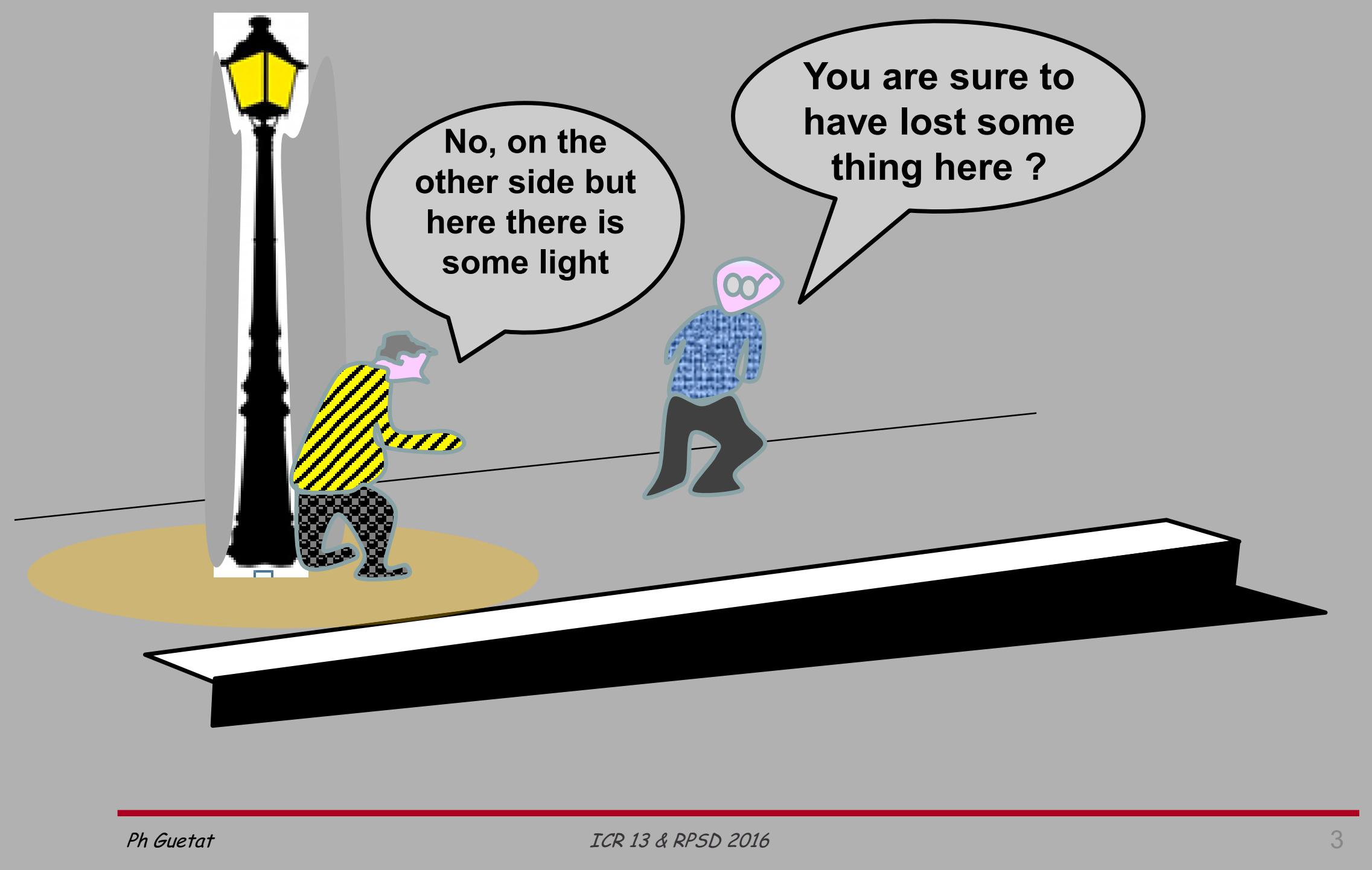




\section{I - REFERENCE VALUES}


- Dose criteria

$0.1 \mathrm{mSv}-1 \mathrm{mSv}-20 \mathrm{mSv}-100 \mathrm{mSv}-0.3 \mathrm{~Sv}-5 \mathrm{~Sv}$

\section{Natural range}

- Dose rate : Sv in 1 year, month, day?

External - internal (inhalation/ ingestion $1^{\text {st }}$ crop \& next crops)

- Specific activity , surface activity.

- Food : in $\mathrm{kBq} / \mathrm{kg}$

- Waste : in $\mathrm{kBq} / \mathrm{kg}$ or Sv. $\mathrm{h}^{-1} \cdot \mathrm{m}^{-2} \quad 4$ Orders of magnitude,

- Evolution with time not black and white, Time is important, Protection margin useful? 
1. Meltdown is necessarily associated to a significant release of energy. Releases are at a big height which brings an important protection to the neighborhood.

2. Delay before release $=$ very short-lived nuclides disappear. Suppress effect on forest by $\beta$.

3. Melting is not explosion : Non-volatile Nuclides not emitted.

- Evacuation induces other more conventional but real dangers.

- The cure can be worse than the disease, especially if the evil is imaginary. 


\section{Cea Reference values as elements of optimization}

Relation between dose \& relative risk for solid cancers and leukemia : Hiroshima Nagasaki
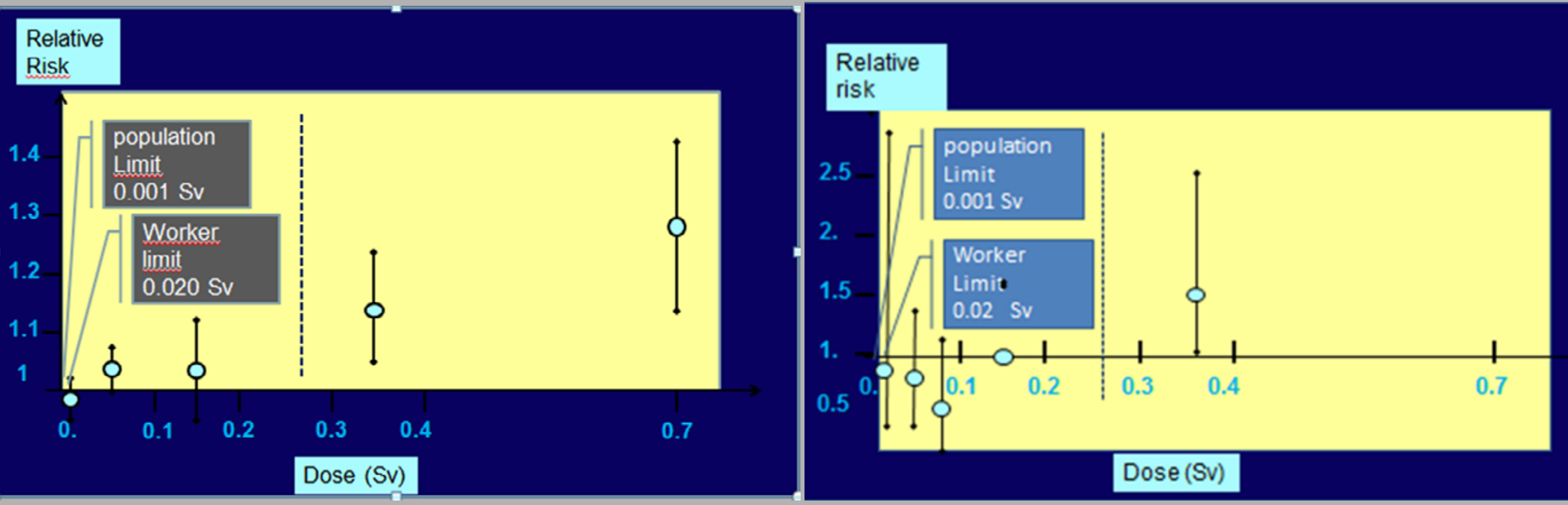

ICRP reference : $0.001,0.02$ and $0.1 \mathrm{~Sv}$

In Japan: 0.3 Sv for workers = a good choice No clinical effect - no detectable stochastic effect 


\section{cea DNA damages short term? (weeks}

\begin{tabular}{|c|c|c|c|}
\hline $\begin{array}{c}\text { DNA Damage to } \\
\text { thyroid cells }\end{array}$ & $\begin{array}{c}\text { spontaneous } \\
\text { over 1 month }\end{array}$ & $\begin{array}{c}\text { Chernobyl } \\
\text { young children }\end{array}$ & $\begin{array}{c}\text { Fukushima } \\
\text { young children }\end{array}$ \\
\hline exposure & - & $\begin{array}{c}\text { 3 Gy (2 and more) } \\
\text { Inhal. \& ingest. }\end{array}$ & Max 0.08 Gy \\
\hline $\begin{array}{c}\text { simple strand } \\
\text { breaks }\end{array}$ & $\begin{array}{c}300000- \\
1700000\end{array}$ & 3000 & 80 \\
\hline & 400000 & & 160 \\
\hline & 100000 & 6000 & $3(1 \%)$ \\
\hline $\begin{array}{c}\text { Nucleobase loss } \\
\text { Bases modification }\end{array}$ & 240 & $120(50 \%)$ & \\
\hline
\end{tabular}

no effect will be visible (exist) in Japan 


\section{cea Reference values for food and feed}

\begin{tabular}{|c|c|c|c|c|c|c|}
\hline $\mathrm{Bq} / \mathrm{kg}$ & $\begin{array}{l}\text { Infant } \\
\text { food }\end{array}$ & $\begin{array}{c}\text { Dairy } \\
\text { produce }\end{array}$ & $\begin{array}{l}\text { Other } \\
\text { major } \\
\text { foods }\end{array}$ & $\begin{array}{c}\text { Liquid } \\
\text { food (CA), } \\
\text { \& water } \\
(W H O)\end{array}$ & $\begin{array}{l}\text { Minor } \\
\text { food }\end{array}$ & Feed \\
\hline I131 & $150 / 100$ & $500 / 100$ & $2000 / 100$ & $125 / 10$ & 20000 & \\
\hline $\begin{array}{l}\text { Cesium } \\
134-137\end{array}$ & $400 / 1000$ & $\begin{array}{c}1000 / \\
1000\end{array}$ & $\begin{array}{c}1250 / \\
1000\end{array}$ & $1000 / 10$ & 12500 & $\begin{array}{l}\text { Pigs............... } 1350 \\
\text { Poultry, lambs, } \\
\text { calves.......... } \quad 2500 \\
\text { Other........... } \quad 5000\end{array}$ \\
\hline Tritium & $-/ 1000$ & $-/ 10000$ & $/ 10000$ & $-/ 10000$ & & $\begin{array}{c}\text { (As Organic } \\
\text { molecules) }\end{array}$ \\
\hline Pu239 & $1 / 1$ & $20 / 10$ & $80 / 10$ & $20 / 1$ & 800 & \\
\hline \multicolumn{7}{|c|}{$\begin{array}{l}\text { 1'rst crop Food ban Fundamental } \\
\text { Sv : very low but today +/- unavoidable. } \\
\text { No reason to choose lower values } \\
\text { laximum, average, 95\%? M= Max/10) }\end{array}$} \\
\hline
\end{tabular}




\begin{tabular}{|c|c|c|c|c|c|}
\hline \multicolumn{6}{|c|}{ Case dry deposition for $1 \mathrm{MBq} \cdot \mathrm{m}^{-2}$ Total deposition } \\
\hline \multirow{4}{*}{$\begin{array}{l}\text { External } \\
\text { irradiation }\end{array}$} & \multirow[t]{2}{*}{ infinite surface } & Cs134 & $5.5 \mu S \cdot h^{-1}$ & 33 mSv.an ${ }^{-1}$ & \multirow{2}{*}{$\begin{array}{l}\mathrm{Bq}{ }^{134} \mathrm{Cs} \&{ }^{137} \mathrm{Cs} \\
\text { not equivalent }\end{array}$} \\
\hline & & Cs137 & 2. $\mu S v \cdot h^{-1}$ & $12{\mathrm{mSv} . \mathrm{an}^{-1}}^{-1}$ & \\
\hline & \multirow{2}{*}{\begin{tabular}{|c|}
$\begin{array}{l}\text { Homogeneous } \\
\text { after ploughing } \\
3 \mathrm{~Bq} / \mathrm{g} \\
\end{array}$ \\
\end{tabular}} & Cs134 & $0.9 \mu S v \cdot h^{-1}$ & $5,3 \mathrm{mSv} \cdot \mathrm{an}^{-1}$ & $\begin{array}{l}\text { Fed. Guid } \mathrm{n}^{\circ} 12 \\
\text { Depth } 30 \mathrm{~cm}\end{array}$ \\
\hline & & Cs137 & $0.33 \mu S v . h^{-1}$ & 2 mSv.an $^{-1}$ & \\
\hline \multirow[t]{2}{*}{ Vegetable } & Foliar deposition & aerosols & $\approx 10^{6} \mathrm{~Bq} \cdot \mathrm{kg}^{-1}$ & existing crop & 1 to $5 \mathrm{~kg}_{\text {Fresh } \mathrm{w}} \cdot \mathrm{m}^{-2}$ \\
\hline & Soil-plant transfer & Cs 134137 & $\begin{array}{c}30-300 \\
\mathrm{~Bq} \cdot \mathrm{kg}^{-1}\end{array}$ & next crops & (Fresh weight) \\
\hline Cow Milk & $\begin{array}{l}\text { feed } \\
\text { contamination }\end{array}$ & Cs 134137 & $\begin{array}{c}1.10^{5} \mathrm{~Bq} \cdot \mathrm{L}^{-1} \\
\max \end{array}$ & short term & (Dynamic model) \\
\hline Cow meat & soil> grass>meat & Cs137 & $\begin{array}{c}50-500 \\
\mathrm{~Bq} \cdot \mathrm{kg}^{-1}\end{array}$ & Long term & \\
\hline Exemption levels & BSS - Transport & Cs 134137 & $10 \mathrm{~Bq} \cdot \mathrm{g}^{-1}$ & Japan 7 Bq. - $^{-1}$ & local/national \\
\hline
\end{tabular}

2 - 3 MBq.m-2 of Cs 137, a physical boundary 
$160 \mu \mathrm{Sv} / \mathrm{h} \quad$ Milk and meat :

feed from

Te elsewhere

$8 \quad$ I ${ }^{134} \mathrm{Cs}$

$$
14 \mu \mathrm{Sv} / \mathrm{h}=90 \mathrm{mSv} \cdot \mathrm{y}^{-1}
$$

plowing $4-5$

$$
2.4 \mu \mathrm{Sv} / \mathrm{h}=14 \mathrm{mSv} \cdot \mathrm{y}^{-1}
$$

Veg, Milk and meat $<1000$ Bq. $\mathrm{kg}^{-1}$ with local feed

\section{${ }^{137}$ Cs}

Radio 3

$4 \mathrm{mSv}^{-\mathrm{y}^{-1}}$ decay

0

1 month

1 year

5 years

2 (or 3?) MBq.m-2 of Cs 137, a consistency in time 


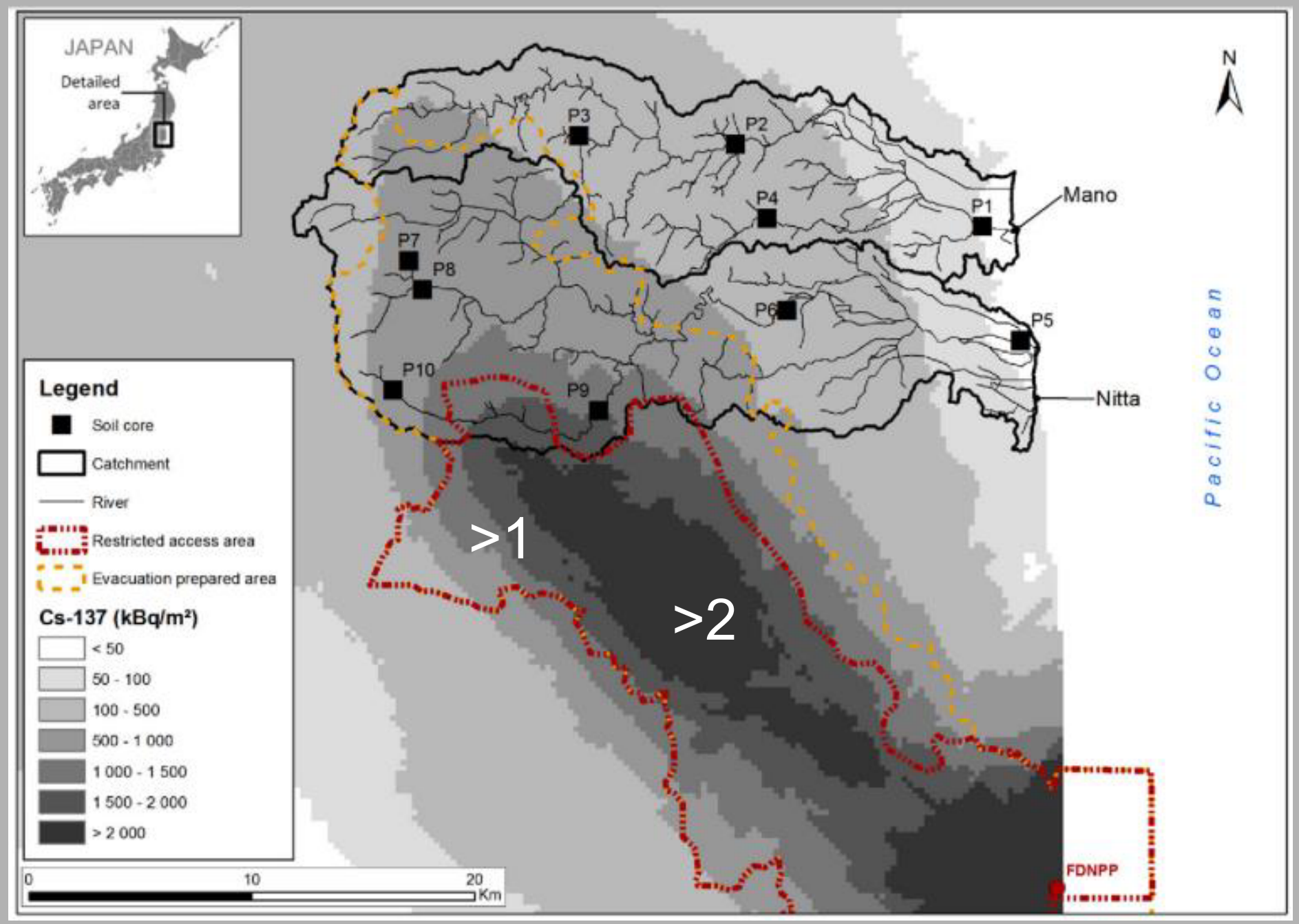




\section{cea Decrease of deposition versus surface}

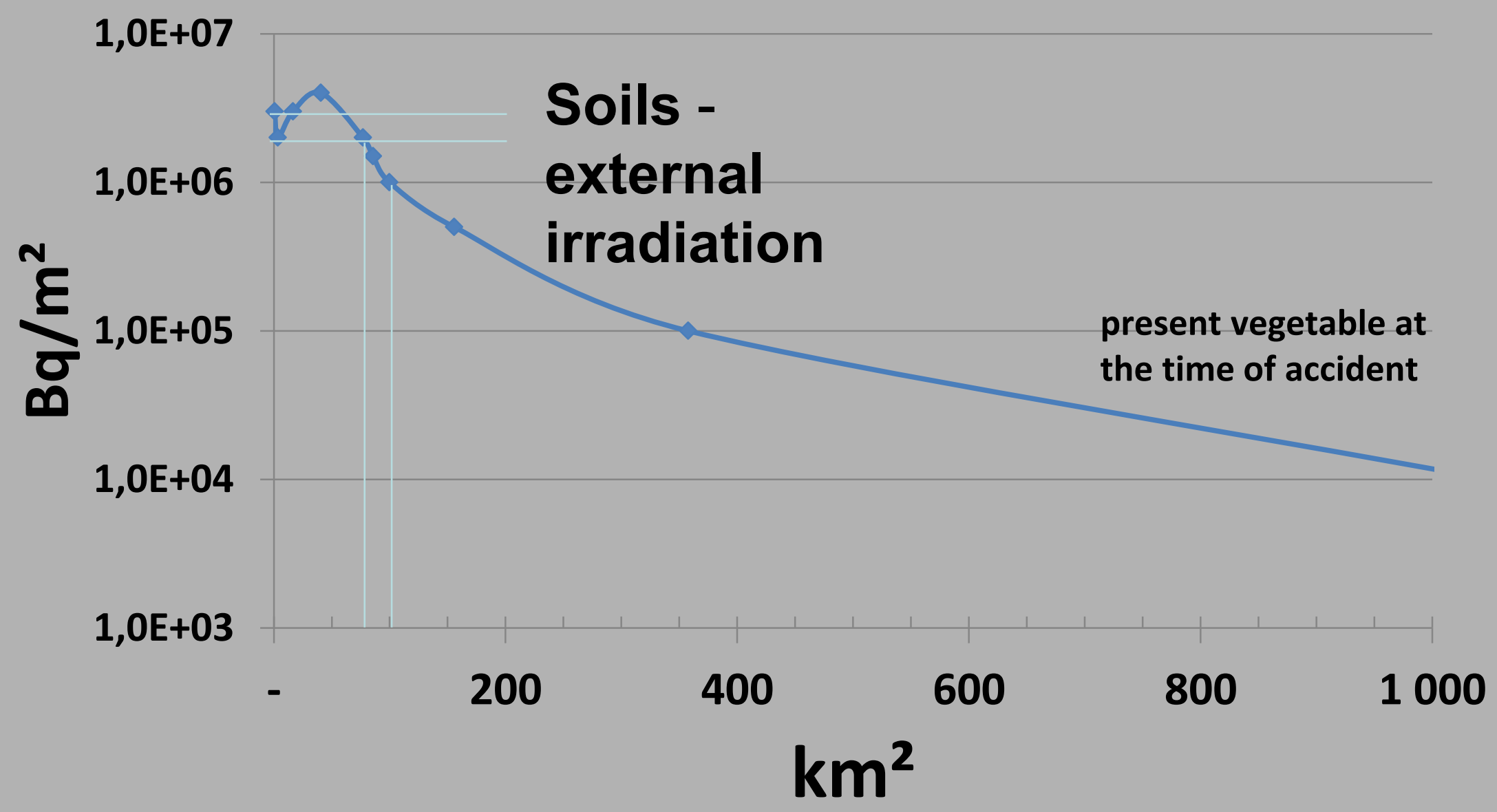


II - WASTE 
- The best waste is the waste that does not exist.

- Soil is a wealth to preserve

- Food is an important but short-term trouble.

\section{To avoid waste}

- Plowing, digging over

- Vegetable grubbing and burial by plowing, digging over.

- Distribution of uncontaminated Feed

- If contaminated milk, disposed on farm by spreading with urines/slurry 


\section{III - OTHER NUCLIDES}


 \\ water}

- Is present in CANDU \& ITER (risk = fire ?)

- water (HTO), organic (in environment, plants), (Gas HT: no risk), metallic hydrides.

- HTO : Very low radiotoxicity - rapid turnover 12 y period

- Very quick decrease in plants in 2 days (100 to 1000).

- No bioaccumulation, no bioconcentration. (major element)

- No health risk, but psychological risk.

- Food ban for some days, for few $\mathrm{km}$ for $10 \mathrm{~g} \mathrm{~T}$.

- Reference value $10^{4} \mathrm{~Bq} / \mathrm{kg}$ far too low at short term. Should be $10^{6} 10^{7} \mathrm{~Bq} / \mathrm{kg}$ for intervention.

- Dilution is absolutely the best, (especially in ocean). 
- Fuel reprocessing

- Dense metal, non-volatile, no external irradiation, non-metabolized element.

- Transfer to plants and animals very small.

- Resuspension is of minor importance.

- For a standard $1 \mathrm{~g}$ release, food restriction for leaf vegetable on few $\mathrm{km}$.

- Incineration is possible but useless.

- Standard impact assessment much over estimated. (density, veg. washing, dose factors) 
- A major nuclear accident (with food ban) has presently no health effect, and environmental/waste effects are mainly psychological. (LNT law)

- Consistency between the criteria is necessary.

- Dilution has to be a large part of the postaccidental management.

- Food and Feed ban can be done at large scale. Soils should be preserved.

- Experts should not stress population and decision makers. 